\title{
Polar Phonons in Relaxor Ferroelectric 0.2PSN-0.4PMN-0.4PZN
}

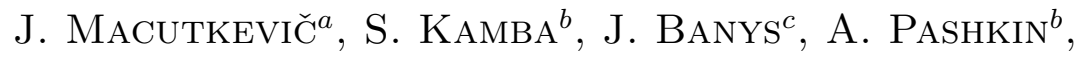 \\ K. Bormanis ${ }^{d}$ And A. SternberG ${ }^{d}$ \\ ${ }^{a}$ Semiconductor Physics Institute, A. Goštauto 11, Vilnius, Lithuania \\ ${ }^{b}$ Institute of Physics, ASCR, Na Slovance 2, Prague 8, Czech Republic \\ ${ }^{c}$ Faculty of Physics, Vilnius University, Sauletekio 9, Vilnius, Lithuania \\ ${ }^{d}$ Institute of Solid State Physics, University of Latvia \\ 8 Kengaraga str., Riga, Latvia
}

\begin{abstract}
Relaxor ferroelectrics $\quad 0.2 \mathrm{PbSc}_{1 / 2} \mathrm{Nb}_{1 / 2} \mathrm{O}_{3}-0.4 \mathrm{PbMg}_{1 / 3} \mathrm{Nb}_{2 / 3} \mathrm{O}_{3}-$ $0.4 \mathrm{PbZn}_{1 / 3} \mathrm{Nb}_{2 / 3} \mathrm{O}_{3}$ ceramics were studied by means of the Fourier transform infrared reflection and $\mathrm{THz}$ transmission spectroscopy in the temperature range of $20-500 \mathrm{~K}$. On heating from low temperatures, the $A_{1}$ component of the strongly split $\mathrm{TO}_{1}$ mode softens towards the Burns temperature, but the softening ceases near $400 \mathrm{~K}$, which could be a signature of polar cluster percolation temperature.
\end{abstract}

PACS numbers: 78.30.-j, 63.20.-e, 71.55.Jv

\section{Introduction}

Solution of relaxor enigma is still one of the most challenging problems in the physics of ferroelectrics. Since the discovery of the archetypical relaxor material $\mathrm{PbMg}_{1 / 3} \mathrm{Nb}_{2 / 3} \mathrm{O}_{3}$ (PMN) [1], the origin of its key features - (i) anomalous broad dielectric dispersion at low temperatures, (ii) non-ergodic behavior, and (iii) virtually no symmetry breaking after zero field cooling down to the lowest temperatures - has been interpreted controversially. No breaking of cubic symmetry was observed at any temperature in PMN in absence of bias electric field [2]. However, for $\mathrm{PbSc}_{1 / 2} \mathrm{Nb}_{1 / 2} \mathrm{O}_{3}(\mathrm{PSN})$ and $\mathrm{PbZn}_{1 / 3} \mathrm{Nb}_{2 / 3} \mathrm{O}_{3}(\mathrm{PZN})$ a ferroelectric phase transition from cubic to rhombohedral phase was reported near $378 \mathrm{~K}$ [3] and $410 \mathrm{~K}$ [4], respectively. Ternary solid solutions of PSN-PZN-PMN relaxor ferroelectrics have been first synthesized and investigated by Dambekalne et al. [5]. Results of broadband dielectric spectroscopy of some PMN-PSN-PZN ceramics have been already published [6].

The aim of the present work is to investigate infrared (IR) and THz spectra of $0.2 \mathrm{PSN}-0.4 \mathrm{PMN}-0.4 \mathrm{PZN}$ relaxor ceramics with emphasis on discussion of activities and temperature dependences of optic phonons in IR spectra. 


\section{Experimental}

The ternary 0.2PSN-0.4PMN-0.4PZN solid solution was synthesized by solid state reactions from high grade oxides $\mathrm{PbO}_{3}, \mathrm{Nb}_{2} \mathrm{O}_{5}, \mathrm{MgO}, \mathrm{ZnO}, \mathrm{Sc}_{2} \mathrm{O}_{3}$ [5]. Measurements at $\mathrm{THz}$ frequencies from $3 \mathrm{~cm}^{-1}$ to $30 \mathrm{~cm}^{-1}$ (90-900 $\mathrm{GHz}$ ) were performed in the transmission mode using a time-domain $\mathrm{THz}$ spectrometer based on an amplified femtosecond laser system. IR reflectivity spectra were obtained using a Fourier transform IR (FTIR) spectrometer Bruker IFS 113v in the frequency range of $20-3300 \mathrm{~cm}^{-1}(0.6-100 \mathrm{THz})$ above room temperature, at lower temperature the reduced spectral range up to $650 \mathrm{~cm}^{-1}$ was investigated because this is the transparency region of polyethylene windows in the cryostat (Oxford Inst.).

\section{Results and discussion}

IR and $\mathrm{THz}$ reflectivity spectra of $0.2 \mathrm{PSN}-0.4 \mathrm{PMN}-0.4 \mathrm{PZN}$ ceramics taken at various temperatures are shown in Fig. 1. Reflectivity spectra below $30 \mathrm{~cm}^{-1}$ were calculated from $\mathrm{THz}$ transmission spectra. The spectral range above $800 \mathrm{~cm}^{-1}$ is not shown because the reflectivity is almost flat at higher frequencies, approaching the value given by the high-frequency permittivity $\varepsilon_{\infty}$. IR reflectivity

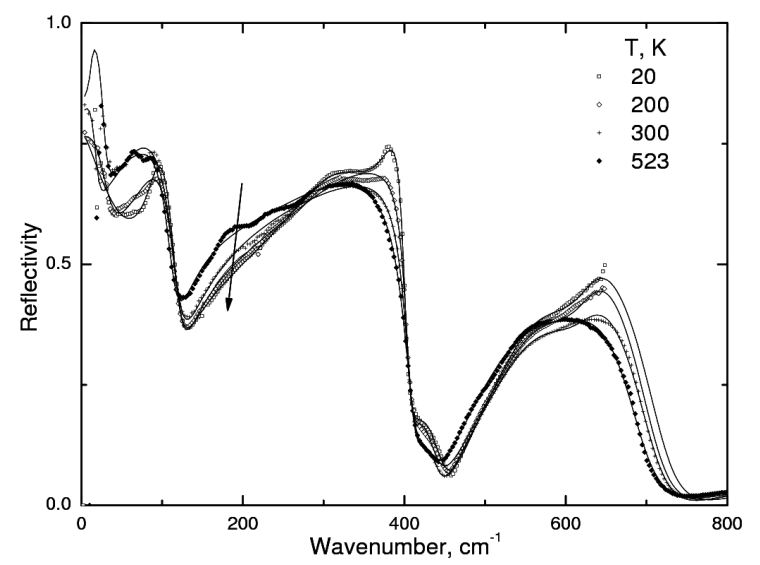

Fig. 1. IR and THz reflectivity spectra of $0.2 \mathrm{PSN}-0.4 \mathrm{PMN}-0.4 \mathrm{PZN}$ ceramics at various temperatures. Solid lines are results of the fits.

and $\mathrm{THz}$ dielectric spectra were fitted simultaneously, using a generalized-oscillator model of the factorized form of the complex permittivity:

$$
\varepsilon^{*}(\omega)=\varepsilon^{\prime}(\omega)-\mathrm{i} \varepsilon^{\prime \prime}(\omega)=\varepsilon_{\infty} \prod_{j} \frac{\omega_{\mathrm{LO} j}-\omega^{2}+\mathrm{i} \omega \gamma_{\mathrm{LO} j}}{\omega_{\mathrm{TO} j}-\omega^{2}+\mathrm{i} \omega \gamma_{\mathrm{TO} j}},
$$

where $\omega_{\mathrm{TO} j}$ and $\omega_{\mathrm{LO} j}$ mark the transverse (TO) and longitudinal (LO) frequency of the $j$-th mode, respectively, and $\gamma_{\mathrm{TO} j}$ and $\gamma_{\mathrm{LO} j}$ denote their corresponding damping constants. $\varepsilon^{*}(\omega)$ is related to reflectivity $R(\omega)$ by 


$$
R(\omega)=\left|\frac{\sqrt{\varepsilon^{*}(\omega)}-1}{\sqrt{\varepsilon^{*}(\omega)}+1}\right| .
$$

Real and imaginary parts of $\varepsilon^{*}(\omega)$ obtained from the fits of IR reflectivity and $\mathrm{THz}$ dielectric spectra are shown in Fig. 2. Eight polar phonons were resolved in the fits of reflectivity below $150 \mathrm{~K}$ and seven modes at higher temperatures. Also

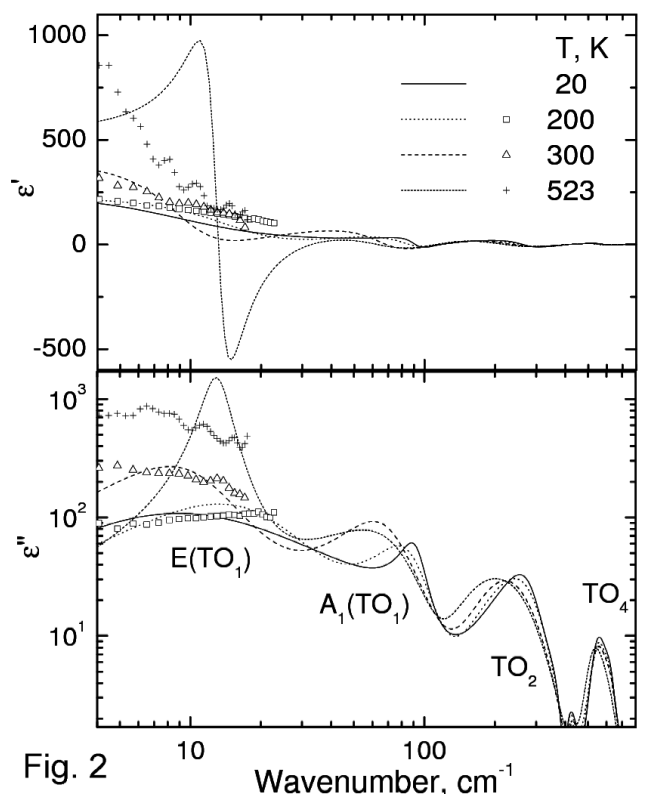

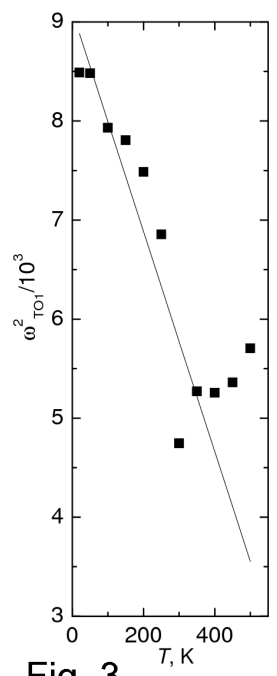

Fig. 3

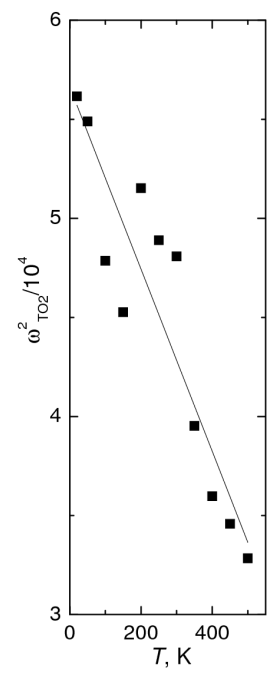

Fig. 2. Complex dielectric permittivity obtained from the fit of IR and THz spectra of $0.2 \mathrm{PSN}-0.4 \mathrm{PMN}-0.4 \mathrm{PZN}$ ceramics.

Fig. 3. Cochran fit to $A_{1}\left(\mathrm{TO}_{1}\right)$ and $\mathrm{TO}_{2}$ modes in $0.2 \mathrm{PSN}-0.4 \mathrm{PMN}-0.4 \mathrm{PZN}$.

interesting phonon anomalies were observed. Most of phonon frequencies exhibit softening up on heating. The most remarkable softening is seen for the lowest frequency $A_{1}\left(\mathrm{TO}_{1}\right)$ phonon (see Fig. 3). Similar soft $\mathrm{TO}_{1}$ mode (the Last mode expressing predominantly the vibration of rigid $\mathrm{BO}_{6}$ octahedra against $\mathrm{Pb}$ atoms) was observed in PMN and PST [7-10]. This mode was explained as a ferroelectric soft mode in polar clusters [7], which softens close to the Burns temperature. The soft mode frequency follows the Cochran law:

$$
\omega_{\mathrm{SM}}^{2}=A\left(T_{\mathrm{cr}}-T\right),
$$

where the constant $A=11.1 \mathrm{~cm}^{2} / \mathrm{K}$ and $T_{\text {cr }}=820 \mathrm{~K}$ is the critical softening temperature. It is really difficult to assert that this temperature corresponds to the Burns temperature, since the experimental $\mathrm{TO}_{1}$ frequencies start to increase already above $400 \mathrm{~K}$. We speculate that the $T_{l}$ temperature corresponds to the temperature, below which the polar clusters are percolated. The effective soft mode cannot completely soften in the system of non-percolated clusters [11] due 
to rise of the effective soft phonon frequency in the composite of polar clusters with non-polar matrix. Therefore, the leveling off (or even increase) of the soft mode frequency is seen above $T_{l} \approx 400 \mathrm{~K}$.

Surprisingly, the $\mathrm{TO}_{2}$ polar mode frequency also softens on heating and follows the Cochran law (see Fig. 3). The $T_{\text {cr }}$ of $\mathrm{TO}_{2}$ mode is equal to $1231 \mathrm{~K}$ and $A=45.97 \mathrm{~cm}^{2} / \mathrm{K}$. This mode is the so-called Slater mode describing predominantly vibration of $\mathrm{B}$ atoms against oxygen octahedral [10]. The B sites exhibit large chemical disorder (4 various atoms of different valency!) and the temperature $T_{\text {cr }}$ of $\mathrm{TO}_{2}$ modes can correspond to temperature, at which the $\mathrm{B}$ site atoms could migrate, because its value is close to a melting point.

\section{Acknowledgments}

This work was supported by the Grant Agency of the Czech Republic (Projects Nos. 202/06/0403). J.M. is supported by the project BPD 2004-ESF2.5.0-05/0029.

\section{References}

[1] G.A. Smolenskii, A.I. Agranovskaya, Sov. Phys.-Tech. Phys. 3, 1380 (1958).

[2] N. de Mathan, E. Husson, G. Calvarin, J.R. Gavari, A.W. Hewat, A. Morell, J. Phys., Condens. Matter 3, 8159 (1991).

[3] C. Perrin, N. Menguy, E. Suard, Ch. Muller, C. Caranoni, A. Stepanov, J. Phys., Condens. Matter 12, 7523 (2000).

[4] E.H. Kisi, J.S. Forester, J. Phys., Condens. Matter 17, L381 (2005).

[5] M. Dambekalne, K. Bormanis, A. Sternberg, I. Brante, Ferroelectrics 240, 221 (2000).

[6] J. Macutkevic, S. Kamba, J. Banys, A. Brilingas, A. Pashkin, J. Petzelt, K. Bormanis, A. Sternberg, Phys. Rev. B 74, 104106 (2006).

[7] V. Bovtun, S. Kamba, A. Pashkin, M. Savinov, P. Samoukhina, J. Petzelt, Ferroelectrics 298, 23 (2004).

[8] S. Kamba, M. Kempa, V. Bovtun, J. Petzelt, K. Brinkman, N. Setter, J. Phys., Condens. Matter 17, 3965 (2005).

[9] S. Kamba, M. Berta, M. Kempa, J. Petzelt, K. Brinkman, N. Setter, J. Appl. Phys. 98, 074103 (2005).

[10] J. Hlinka, J. Petzelt, S. Kamba, D. Noujni, T. Ostapchuk, Phase Transit. 79, 41 (2006).

[11] I. Rychetsky, J. Petzelt, Ferroelectrics 303, 137 (2004). 